OPEN ACCESS

Edited by:

Guido Moll,

Charité - Universitätsmedizin Berlin,

Germany

Reviewed by:

Federica Casiraghi,

Mario Negri Institute

of Pharmacological Research, Italy

Selim Kuci,

University Hospital Frankfurt,

Germany

${ }^{*}$ Correspondence:

Marc Schmitz

marc.schmitz@tu-dresden.de

Specialty section:

This article was submitted to

Stem Cell Research,

a section of the journal

Frontiers in Cell and Developmental

Biology

Received: 04 December 2020 Accepted: 19 January 2021

Published: 09 February 2021

Citation:

Müller L, Tunger A, Wobus $M$, von Bonin $M$, Towers $R$,

Bornhäuser M, Dazzi F, Wehner R and Schmitz M (2021) Immunomodulatory

Properties of Mesenchymal Stromal

Cells: An Update.

Front. Cell Dev. Biol. 9:637725.

doi: 10.3389/fcell.2021.637725

\section{Immunomodulatory Properties of Mesenchymal Stromal Cells: An Update}

\author{
Luise Müller', Antje Tunger1,2, Manja Wobus ${ }^{3}$, Malte von Bonin'2,3,4, Russell Towers ${ }^{3}$, \\ Martin Bornhäuser ${ }^{2,3,4,5}$, Francesco Dazzi ${ }^{6}$, Rebekka Wehner ${ }^{1,2,4}$ and Marc Schmitz 1,2,4,5* \\ ${ }^{1}$ Institute of Immunology, Faculty of Medicine Carl Gustav Carus, TU Dresden, Dresden, Germany, ${ }^{2}$ National Center \\ for Tumor Diseases (NCT), Partner Site Dresden, Dresden, Germany, ${ }^{3}$ Department of Medicine I, University Hospital Carl \\ Gustav Carus, TU Dresden, Dresden, Germany, ${ }^{4}$ German Cancer Consortium (DKTK), Partner Site Dresden, and German \\ Cancer Research Center (DKFZ), Heidelberg, Germany, ${ }^{5}$ Center for Regenerative Therapies Dresden (CRTD), TU Dresden, \\ Dresden, Germany, ${ }^{6}$ School of Cancer and Pharmacological Sciences and KHP Cancer Research UK Centre, King's \\ College London, London, United Kingdom
}

Mesenchymal stromal cells (MSCs) are characterized by an extraordinary capacity to modulate the phenotype and functional properties of various immune cells that play an essential role in the pathogenesis of inflammatory disorders. Thus, MSCs efficiently impair the phagocytic and antigen-presenting capacity of monocytes/macrophages and promote the expression of immunosuppressive molecules such as interleukin (IL)-10 and programmed cell death 1 ligand 1 by these cells. They also effectively inhibit the maturation of dendritic cells and their ability to produce proinflammatory cytokines and to stimulate potent T-cell responses. Furthermore, MSCs inhibit the generation and proinflammatory properties of $\mathrm{CD}^{+}{ }^{+} \mathrm{T}$ helper $(\mathrm{Th}) 1$ and Th17 cells, while they promote the proliferation of regulatory $T$ cells and their inhibitory capabilities. MSCs also impair the expansion, cytokine secretion, and cytotoxic activity of proinflammatory CD8 ${ }^{+} \mathrm{T}$ cells. Moreover, MSCs inhibit the differentiation, proliferation, and antibody secretion of $\mathrm{B}$ cells, and foster the generation of $\mathrm{IL}-10$-producing regulatory $\mathrm{B}$ cells. Various cell membrane-associated and soluble molecules essentially contribute to these MSCmediated effects on important cellular components of innate and adaptive immunity. Due to their immunosuppressive properties, MSCs have emerged as promising tools for the treatment of inflammatory disorders such as acute graft-versus-host disease, graft rejection in patients undergoing organ/cell transplantation, and autoimmune diseases.

Keywords: mesenchymal stromal cells, immunomodulation, macrophages, dendritic cells, $\mathrm{T}$ cells

\section{INTRODUCTION}

Mesenchymal stromal cells (MSCs) are characterized in vitro by the adherence to plastic surfaces, the expression of CD73, CD90, and CD105, the lack of expression of the hematopoietic and endothelial markers CD11b, CD14, CD19, CD34, CD45, CD79a, and detectable amounts of human leukocyte antigen (HLA)-DR as well as the capability to differentiate into adipocytes, chondrocytes, and osteoblasts (Dominici et al., 2006; Viswanathan et al., 2019). Additionally, the MSC committee of the International Society for Cell and Gene Therapy recommends to support 
the phenotypical characterization by functional assays confirming hallmark properties of MSCs such as secretion of soluble factors and immunomodulation (Viswanathan et al., 2019). While MSCs were first isolated from bone marrow (BM), a variety of tissues were found to harbor MSCs comprising adipose, placenta, umbilical cord (UC), dental pulp, and other tissues. Due to their accessibility, adipose tissue-derived (AD)MSCs and UC-MSCs have gained an increasing popularity, especially for clinical studies. Even though all MSCs share certain characteristics, the discrimination between MSCs of different origins became particularly important since several studies have found striking differences, regarding not only their marker expression and cytokine profile but also their functional properties (Kozlowska et al., 2019; Ritter et al., 2019; Ménard et al., 2020; Petrenko et al., 2020; Song et al., 2020). For example, BM-MSCs secreted the highest amount of proangiogenic interleukin (IL)-8 and vascular endothelial growth factor compared to MSCs derived from adipose tissue, skeletal muscle, and skin, while AD-MSCs displayed the strongest ability to secrete IL-6 (Kozlowska et al., 2019). Additionally, BM-MSCs were found to have the most prominent immunosuppressive capacities in both cell contact-dependent and paracrine settings (Petrenko et al., 2020). Despite several challenges, MSCs have gained increasing attention in recent years. Their differentiation capability allows for their therapeutic use in regenerative medicine and tissue engineering. In addition, MSCs exhibit a low immunogenicity and display an extraordinary capacity to modulate immune responses. While these traits make MSCs attractive candidates for the treatment of immune-related disorders like autoimmune diseases, acute graft-versus-host disease (aGvHD), and sepsis, their modulatory action strongly depends on the environmental stimuli (Wang et al., 2016). It has been shown that under certain conditions, MSCs can promote immune responses by secreting proinflammatory cytokines and acting as antigen-presenting cells. Their immunostimulatory capabilities can be converted into an immunosuppressive phenotype by a process termed "licensing." This phenotypic and functional shift is mediated by inflammatory cytokines such as interferon (IFN) $-\gamma$ or tumor necrosis factor (TNF)- $\alpha$ (Krampera, 2011). The dual role of MSCs should be considered when assessing their immunomodulatory capacities and their use in clinical applications (Carvalho et al., 2019). Here, we focus on recent studies exploring the impact of MSCs on the phenotype and functional properties of monocytes/macrophages, dendritic cells (DCs), T cells, and B cells that play a major role in various immune-driven disorders. Furthermore, the cell membrane-associated and soluble molecules that contribute to the immunomodulatory effects of MSCs are summarized.

\section{MODULATION OF INNATE IMMUNITY BY MSCs}

\section{Monocytes/Macrophages}

Macrophages are important components of innate immunity and play an important role in the pathogenesis of various immune-mediated diseases. Based on their phenotype and functional properties, macrophages can be classified into proinflammatory M1 and anti-inflammatory M2 macrophages (Shapouri-Moghaddam et al., 2018). Recently, it has been demonstrated that MSCs efficiently promote macrophage polarization toward the M2 type, which is considered to be beneficial in immune-driven disorders. This M2 polarizing effect of MSCs is mediated by various soluble molecules including prostaglandin E2 (PGE2) (Németh et al., 2009; Vasandan et al., 2016), indolamin-2,3-dioxygenase (IDO) (François et al., 2012), IL-6, hepatocyte growth factor (HGF) (Deng et al., 2016), IL-1 receptor antagonist (IL-1RA) (Luz-Crawford et al., 2016), tumor necrosis factor-inducible gene 6 protein (TSG6) (Ko et al., 2016), and transforming growth factor (TGF)- $\beta$ (Liu et al., 2019a). Several studies suggest a key role of PGE2, as the inhibition of PGE2 or the cyclooxygenase-2 (COX2) pathway abrogated the observed inhibitory effects (Németh et al., 2009; Jin et al., 2019; Ortiz-Virumbrales et al., 2020). Previous studies using $\mathrm{AD}$ - or BM-MSCs indicated that the paracrine action is partially mediated by exosomes (Biswas et al., 2019; Cho et al., 2019; He et al., 2019; Liu et al., 2019b; Wang et al., $2020 b)$. The exosomes contained TGF- $\beta$, C1q, semaphorins, and micro (mi) RNAs, regulating the macrophage polarization and inducing overexpression of programmed cell death 1 ligand 1 (PD-L1). Moreover, MSCs can alter the macrophage phenotype by manipulating their metabolic properties, such as glycolysis (Vasandan et al., 2016; Deng et al., 2020).

The MSC-induced M2 polarization is accompanied by an increased secretion of anti-inflammatory IL-10 and Arginase1 and a reduced production of proinflammatory cytokines like TNF- $\alpha$, IL-12, and IL-1 $\beta$ (Kim and Hematti, 2009; Yang et al., 2020). Additionally, exosomes from UC-MSCs reduced the expression of the NLR family pyrin domain containing 3 (NLRP3) inflammasome and involved downstream factors like caspase-1, IL-1 $\beta$, and IL-6 in lipopolysaccharide (LPS)-stimulated macrophages (Jiang et al., 2019). Another study revealed that the effect of IL-10 production by murine MSC-primed macrophages is particularly important, as the depletion of IL-10 by antibodies abrogated beneficial effects of MSC treatment in a model of sepsis (Németh et al., 2009). M2 macrophages, characterized by reduced expression of costimulatory molecules and elevated secretion of anti-inflammatory cytokines like TGF- $\beta$, profoundly inhibit $\mathrm{T}$ cell responses and induce regulatory $\mathrm{T}$ cells (Tregs) (Savage et al., 2008; Schmidt et al., 2016), leading to further immunosuppression and supporting the positive effects of MSC therapy. MSCs also impair both the differentiation and effector function of monocytes. Maqbool et al. (2020) discovered a reduced expression of HLA-DR/DP/DQ and CD86 by monocytes and macrophages upon co-culture with human UC-MSCs. Functional analysis of this interaction revealed a significantly decreased phagocytic capacity and antigen-presenting ability of monocytes and macrophages when co-cultured with UCMSCs. Furthermore, we investigated the impact of MSCs on various immunomodulatory properties of 6-sulfo LacNAc monocytes (slanMo), representing a subset of pro-inflammatory $\mathrm{CD} 14^{-} \mathrm{CD} 16^{+}$non-classical monocytes, which may contribute to the pathogenesis of various inflammatory diseases (Ahmad et al., 2019). We found that MSCs profoundly suppress the 
capacity of slanMo to secrete TNF- $\alpha$, IL-6, and IL-12, improve their IL-10 production, and efficiently inhibit the slanMoinduced proliferation of $\mathrm{CD}^{+}$and $\mathrm{CD}^{+} \mathrm{T}$ cells (Wehner et al., 2009). Additionally, de Witte et al. (2018) demonstrated that upon their phagocytosis by monocytes, UC-MSCs induce a $\mathrm{CD} 14^{++} \mathrm{CD} 16^{+} \mathrm{CD} 206^{+}$phenotype accompanied by increased IL-10 secretion and PD-L1 expression in mice. Further studies revealed that the phagocytosis of cord tissue (CT)-derivedMSCs is mediated by lipoprotein receptor-related proteins on monocytes and macrophages (Min et al., 2020). Moreover, the macrophage reprogramming upon phagocytosis of CTMSCs was shown to be dependent on cytoplasmic RNA processing bodies ( $\mathrm{p}$-bodies), as p-body-deficient MSCs failed to suppress inflammation. P-bodies are membrane-less organelles that contain RNA, miRNA, and proteins which may facilitate macrophage polarization upon phagocytosis of MSCs (Kulkarni et al., 2010). We have discovered similar mechanisms, in which the immunosuppressive effects of MSCs depend on their apoptosis induced by recipient cytotoxic immune effector cells (Galleu et al., 2017; Cheung and Dazzi, 2018). Remarkably, the observed cytotoxicity differed significantly between clinical responders and non-responders to MSC therapy. Subsequently, apoptotic MSCs were shown to induce IDO production by recipient phagocytic cells in a GvHD mouse model and both the depletion of phagocytes and blockade of IDO reduced the beneficial effects.

\section{Dendritic Cells}

Activated DCs display a unique capacity to induce T-cell responses and are the main producers of proinflammatory cytokines (Steinman and Banchereau, 2007; Qian and Cao, 2018). Due to these functional properties, DCs can essentially contribute to the immunopathogenesis of various disorders like aGvHD and autoimmune diseases. Their crucial role makes DCs attractive targets for immune-modulating therapies (Ganguly et al., 2013; Obregon et al., 2017). MSCs can efficiently inhibit the differentiation of DCs from hematopoietic stem cells and monocytes (Nauta et al., 2006; Ramasamy et al., 2007). The latter is facilitated by the downregulation of Cyclin D2, hindering monocytes from entering the G1 phase of the cell cycle. Additionally, MSCs impair the maturation of DCs, reducing their capacity to activate $\mathrm{T}$ cells. This is accompanied by a decreased expression of HLA-DR, CD40, OX40L, CD80, CD83, and CD86 (Jiang et al., 2005; Dong et al., 2018), as well as an increased PD-L1 expression (Lu et al., 2020). Moreover, MSCs shift the cytokine profile of DCs from proinflammatory toward immunoregulatory. For example, a decreased secretion of proinflammatory cytokines by $\mathrm{CD} \mathrm{c}^{+} \mathrm{DCs}$ and an enhanced expression of IL-10 secretion by plasmacytoid (p)DCs upon coculture with MSCs have been reported (Aggarwal and Pittenger, 2005). Similarly, the production of IL-12 by monocyte-derived DCs (moDCs) and IFN- $\alpha$ by pDCs was impaired when cocultured with BM- and UC-MSCs, respectively (Jiang et al., 2005; Chen et al., 2019). These effects were mediated by PGE2, TSG6, IL-6, and macrophage colony-stimulating factor (M-CSF) as the inhibition of these molecules abolished the observed effects (Aggarwal and Pittenger, 2005; Jiang et al., 2005; Liu et al., 2014).
Furthermore, murine AD-MSC-derived exosomes inhibited the IL- 6 secretion and improved the release of IL- 10 and TGF- $\beta$ by DCs (Shahir et al., 2020).

MSCs promote the generation of tolerogenic DCs through different mechanisms. In particular, the induction of regulatory DCs seems to be dependent on Notch signaling (Li et al., 2008). The interaction between Jagged1 on BM-MSCs and Notch2 on DCs results in the generation of regulatory DCs (Li et al., 2020b). Furthermore, the MSC-mediated reduction of major histocompatibility complex (MHC) class II molecules, CD86, and CD40 could be mimicked by recombinant Jagged2 and blocked by a Notch inhibitor in a mouse model of acute lung injury (Lu et al., 2020). Besides cell contact-dependent mechanisms, the MSC-mediated generation of regulatory DCs is facilitated by soluble factors. For example, it has been shown that murine BM-MSCs induce the differentiation into regulatory DCs by HGF secretion (Lu et al., 2019). Further functional data revealed that rat MSCs inhibit the maturation of $\mathrm{CD}_{103}{ }^{+} \mathrm{DCs}$, leading to a decreased ability to prime $\mathrm{CD} 8^{+} \mathrm{T}$ cells (Zhang et al., 2020). Additionally, murine DCs that were treated with MSCderived exosomes failed to stimulate T-cell proliferation upon LPS activation (Shahir et al., 2020).

\section{REGULATION OF ADAPTIVE IMMUNITY BY MSCs \\ T Cells}

$\mathrm{CD}^{+} \mathrm{T}$ helper $(\mathrm{Th})$ cells and $\mathrm{CD}^{+}$cytotoxic $\mathrm{T}$ cells (CTLs) play a pivotal role in the immunopathogenesis of aGVHD, autoimmune diseases, and other inflammatory disorders. Activated CTLs efficiently destroy cells and secrete large amounts of proinflammatory cytokines such as TNF- $\alpha$ and IFN- $\gamma$. Stimulated $\mathrm{CD}^{+}{ }^{+} \mathrm{T}$ cells improve the capacity of DCs to induce CTLs by the interaction between CD40 on DCs and CD40 ligand on $\mathrm{CD}^{+}{ }^{+} \mathrm{T}$ lymphocytes. Furthermore, $\mathrm{CD} 4^{+} \mathrm{T}$ cells provide help for the maintenance and expansion of CTLs by secreting cytokines such as IL-2. Previous studies have demonstrated that MSCs can profoundly suppress the proliferation of $\mathrm{CD}^{+}$and $\mathrm{CD}^{+} \mathrm{T}$ cells by both paracrine and cell contact-dependent mechanisms (Di Nicola et al., 2002; Krampera et al., 2003, 2006). While some authors argue that the anti-proliferative effect is not mediated by apoptosis (Krampera et al., 2006; Benvenuto et al., 2007), others reported an increased apoptosis of lymphocytes upon co-culture with MSCs (Zhao et al., 2012). This effect was dependent on the expression of Fas ligand (FasL) as the knockdown of FasL in MSCs by small interfering (si) RNA abrogated the effect on $\mathrm{T}$ lymphocytes as well as their therapeutic impact. As demonstrated by Akiyama et al. (2012), BM-MSCs secrete monocyte chemotactic protein 1 (MCP-1) to recruit $\mathrm{T}$ cells for FasL-mediated apoptosis in a mouse model of systemic sclerosis. More recently, it has been reported that particularly exosomes from murine MSCs mediate the cell cycle arrest of T cells through upregulation of cyclin-dependent kinase inhibitor 1B (CDKN1B) and downregulation of cyclin-dependent kinase 2 (Cdk2) (Lee et al., 2020). Besides the effect on the viability and proliferation of T cells, MSCs can also inhibit the generation and function 
of Th1 and Th17 cells while promoting Th2 cells and Tregs. BM-MSC-educated DCs mediate a shift from Th1 to Th2 and induce Tregs, which is accompanied by a decreased secretion of proinflammatory cytokines like IFN- $\gamma$, IL-17, and IL-6, whereas the production of IL-4 and IL-10 is increased (Wang et al., 2008; Ge et al., 2010). This effect seems to be partially dependent on IDO, as the impact was less prominent in IDO knock-out MSCs. Novel findings indicated that the inhibition of Th17 cells by murine MSCs is also mediated by HGF (Chen et al., 2020). Furthermore, it has been reported that BM-MSCs impair the IL-17 production by Th17 cells in a contact-dependent manner and induce their interconversion into Tregs (Luz-Crawford et al., 2019). They also provided evidence for a novel mechanism, in which MSCs transfer their mitochondria to Th17 cells, and subsequent experiments demonstrated that the artificial transfer of mitochondria impaired their IL-17 production. These findings are supported by another study that showed a mitochondrial transfer from MSCs to $\mathrm{CD}^{+}{ }^{+}$rather than $\mathrm{CD} 8^{+} \mathrm{T}$ cells, which induced increased mRNA levels of FOXP3, CD25, cytotoxic T lymphocyte antigen 4 (CTLA-4), and TGF $\beta 1$ (Court et al., 2020). This subsequently led to the generation of a highly suppressive $\mathrm{CD}_{25}{ }^{+} \mathrm{FoxP}^{+} \mathrm{T}$ cell population. Additionally, exosomes from UC-MSCs inhibited $\mathrm{CD}^{+}$and Th1 cells and reduced their secretion of IFN- $\gamma$ and TNF- $\alpha$ in a mouse model of contact hypersensitivity (Guo et al., 2019). The exosomes also induced Tregs and promoted their IL-10 secretion. Multiple studies demonstrated that MSCs support the expansion and inhibitory capacity of Tregs and foster their generation from conventional T cells (English et al., 2009; Engela et al., 2013; Khosravi et al., 2017). Involved molecules include HGF, PGE2, TGF- $\beta$, and IL-10 (Chen et al., 2020). A recent study confirmed that the promotion of Tregs arises from an epigenetic conversion of conventional $\mathrm{T}$ cells to Tregs rather than expansion of natural Tregs (Azevedo et al., 2020). In a murine model of respiratory infection, UCMSCs were shown to be engulfed by lung phagocytes, which then secreted C-X-C motif ligand (CXCL)9 and CXCL10,

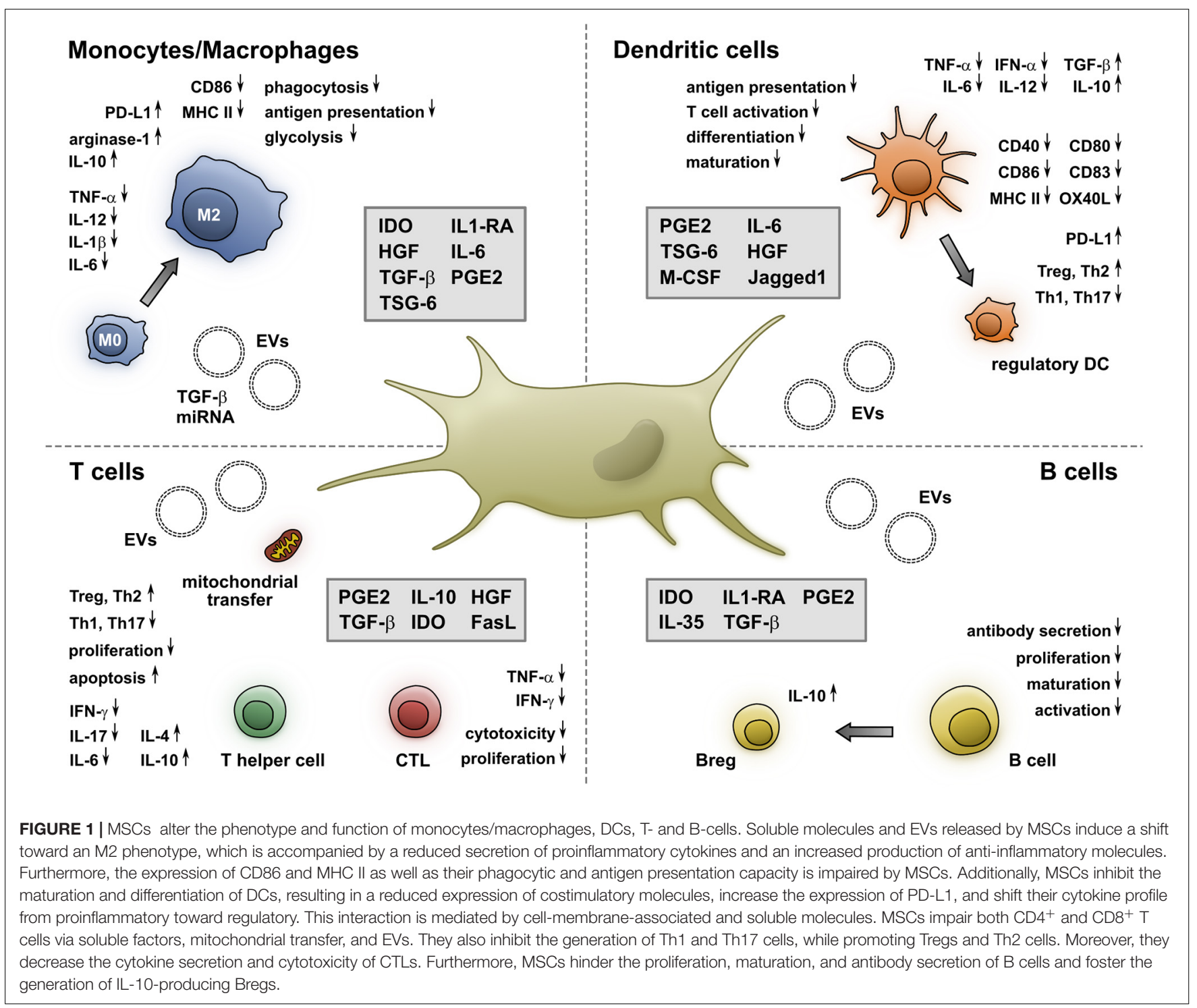


leading to the recruitment of particularly suppressive $\mathrm{C}-\mathrm{X}-\mathrm{C}$ motif receptor (CXCR) $3^{+}$Tregs (Li et al., 2020a). In addition to $\mathrm{T}$ helper cells, MSCs also significantly impair the IFN- $\gamma$ production and cytotoxic activity of CTLs (Maccario et al., 2005; Malcherek et al., 2014).

\section{B Cells}

Activated B lymphocytes produce large amounts of specific antibodies and regulate immune responses by secreting cytokines. Therefore, B cells can crucially contribute to a variety of autoimmune diseases and other antibody-driven pathological mechanisms. MSCs inhibit the proliferation of B cells through an arrest in the G0/G1 phase of the cell cycle (Tabera et al., 2008). Further analyses have shown that MSCs also markedly inhibit the pDC-induced maturation of B cells. Similarly, Magatti et al. (2020) reported a decreased proliferation, maturation, and antibody secretion mediated by MSCs. They also demonstrated that the conditioned medium of MSCs reduces the expression of CD205, CD14, and Toll-like receptor 9 by B cells and that the observed effects are partially mediated by PGE2. A recent study reported that human UC-MSCs impaired the maturation of $\mathrm{B}$ cells by secretion of TGF- $\beta$ (Park et al., 2020). Another important soluble factor is IL1-RA, as MSCs from IL1-RA-deficient mice were unable to inhibit B-cell differentiation (Luz-Crawford et al., 2016). In a mouse model of LPS-induced acute lung injury, MSC treatment blocked the expression of genes involved in chemokine signaling and immunoglobulin expression by $\mathrm{B}$ cells (Feng et al., 2020). While multiple studies support the MSC-induced inhibition of B cell antibody secretion, others argue that MSCs actually promote their antibody production as well as survival, proliferation, and differentiation (Traggiai et al., 2008). The effects of activated MSCs on B cells can vary depending on the level of stimulation. Whenever LPS induced a strong IgG production, this effect was reduced by the addition of MSCs, while a lower LPS-induced IgG secretion was associated with an enhancing effect of MSCs (Rasmusson et al., 2007). Several studies support the differential action of MSCs on B cells, depending on their activation status and the environment. For example, IFN- $\gamma$-primed pediatric MSCs inhibited naïve, memory, and total B cell proliferation, while unprimed MSCs failed to do so (Palomares Cabeza et al., 2019). Similarly, Luk et al. (2017) demonstrated that AD-MSCs only reduce the proliferation and IgG secretion of B cells upon IFN- $\gamma$ exposure, while non-stimulated MSCs induced regulatory B cells (Bregs) and IL-10 production. Further analysis revealed that both interactions are dependent on IDO signaling. Interestingly, the separation of B cells and MSCs in a transwell assay inhibited the suppressive properties of MSCs, suggesting that the involved mechanism requires cell contact or at least close proximity. Another study demonstrated that IL-35 plays a crucial role in the induction of IL-10-producing Bregs by MSCs (Cho et al., 2017). Besides the direct secretion of soluble factors, MSCs also mediate their effects on B cells via extracellular vesicles (EVs). For example, EVs from MSCs that were pretreated to mimic inflammatory conditions inhibit the reorganization of the actin cytoskeleton, which is a crucial event during early B cell activation (Adamo et al., 2019). Furthermore, the treatment of B cells with MSC-derived EVs induced the negative modulation of the PI3K-AKT signaling pathway that is involved in cell proliferation and survival. More recently, it has been demonstrated that MSC-EVs block the interaction between follicular T helper cells and germinal B cells, potentially leading to a reduced GvHD score in a mouse model of chronic GvHD (Guo et al., 2020). In contrast, it has also been reported that the effects of AD-MSCs on B cells are mainly mediated by soluble factors and not EVs (Carreras-Planella et al., 2019).

\section{CONCLUSION}

MSCs display an extraordinary capacity to modulate the phenotype and functional properties of various immune cells (Figure 1). Thus, they excel in inhibiting the maturation and function of DCs and macrophages, while promoting a shift toward regulatory DCs and anti-inflammatory M2 macrophages. They also effectively impair the proliferation and proinflammatory functional properties of B- and T-lymphocytes and induce the generation of Tregs and Bregs. Their immunosuppressive capacities, especially in an inflammatory setting, allow them to efficiently induce clinical responses in patients with immune-mediated disorders (Munneke et al., 2016; Panés et al., 2016; Wang et al., 2016; Bader et al., 2018). Recently, several pre-clinical studies presented promising results in using MSCs or MSC-derived EVs to treat acute lung injury or acute respiratory distress syndrome in Covid-19 patients, and a multitude of clinical studies are currently ongoing (Leng et al., 2020; Moll et al., 2020; Saldanha-Araujo et al., 2020).

However, a significant proportion of patients with immunemediated disorders fail to respond to MSC-based therapy. The heterogeneity and high plasticity of MSCs entail several challenges and might explain the differences in the clinical outcome. For example, recent evidence suggests that $\mathrm{AD}$ MSCs exhibit a stronger immunosuppressive capacity compared to donor-matched BM-MSCs (Ménard et al., 2020). Besides differing origins, additional differences of studies working with MSCs arise from variations in culture conditions and experimental settings. It has been shown that factors like isolation procedure, seeding density, and media composition alter the phenotype of MSCs, emphasizing the need for standardized protocols (Bara et al., 2014; Czapla et al., 2019; Wang et al., 2020a). Another approach to deal with heterogeneous results in clinical trials is the use of biomarkers to predict the clinical response and pre-select superior donor MSCs and eligible patients. For example, TSG6 expression was shown to be a positive predictor of MSC efficacy in a mouse model of sterile inflammation (Lee et al., 2014). While the ideal biomarker would comprise a univariable marker by which MSCs can be selected prior to therapy, this is unlikely to be achieved due to the extraordinary plasticity of MSCs. Instead, mechanistic and functional evaluation may yield better results, such as the level of cytotoxicity by recipient $\mathrm{T}$ and natural killer cells toward MSCs which differed significantly between clinical responders and nonresponders in a study with GvHD patients (Galleu et al., 2017). 
To circumvent the need for cytotoxic actions from recipient cells, the use of apoptotic MSCs proposes another attractive approach and decreases the potential risk of a contribution to tumorigenesis by living MSCs (Weiss and Dahlke, 2019). The cryopreservation required for on demand solutions of viable cells is yet another challenge, as it was shown to influence both phenotypic and functional characteristics of MSCs (Moll et al., 2016). Interestingly, the IFN- $\gamma$-mediated licensing of MSCs prior to therapy might not only enhance their immunosuppressive properties but also protect from cryo-induced reduction of viability (Carvalho et al., 2019). Furthermore, cell-free therapies using MSC-derived EVs have gained increasing attention, since they are easier to generate and administer in large quantities, more suitable for long-term storage, and easier to handle in terms of safety and quality control (Phinney and Pittenger, 2017).

\section{REFERENCES}

Adamo, A., Brandi, J., Caligola, S., Delfino, P., Bazzoni, R., Carusone, R., et al. (2019). Extracellular vesicles mediate mesenchymal stromal cell-dependent regulation of B Cell PI3K-AKT signaling pathway and actin cytoskeleton. Front. Immunol. 10:446. doi: 10.3389/fimmu.2019.00446

Aggarwal, S., and Pittenger, M. F. (2005). Human mesenchymal stem cells modulate allogeneic immune cell responses. Blood 105, 1815-1822. doi: 10. 1182/blood-2004-04-1559

Ahmad, F., Döbel, T., Schmitz, M., and Schäkel, K. (2019). Current concepts on 6-sulfo LacNAc expressing monocytes (slanMo). Front. Immunol. 10:948. doi: 10.3389/fimmu.2019.00948

Akiyama, K., Chen, C., Wang, D., Xu, X., Qu, C., Yamaza, T., et al. (2012). Mesenchymal-stem-cell-induced immunoregulation involves FASLigand-/FAS-mediated T cell Apoptosis. Cell Stem Cell 10, 544-555. doi: 10. 1016/j.stem.2012.03.007

Azevedo, R. I., Minskaia, E., Fernandes-Platzgummer, A., Vieira, A. I. S., da Silva, C. L., Cabral, J. M. S., et al. (2020). Mesenchymal stromal cells induce regulatory $\mathrm{T}$ cells via epigenetic conversion of human conventional CD4 T cells in vitro. Stem Cells 38, 1007-1019. doi: 10.1002/stem.3185

Bader, P., Kuçi, Z., Bakhtiar, S., Basu, O., Bug, G., Dennis, M., et al. (2018). Effective treatment of steroid and therapy-refractory acute graft-versus-host disease with a novel mesenchymal stromal cell product (MSC-FFM). Bone Marrow Transplant. 53:852. doi: 10.1038/s41409-018-0102-z

Bara, J. J., Richards, R. G., Alini, M., and Stoddart, M. J. (2014). Concise review: bone marrow-derived mesenchymal stem cells change phenotype following in vitro culture: implications for basic research and the clinic. Stem Cells 32, 1713-1723. doi: 10.1002/stem.1649

Benvenuto, F., Ferrari, S., Gerdoni, E., Gualandi, F., Frassoni, F., Pistoia, V., et al. (2007). Human mesenchymal stem cells promote survival of T cells in a quiescent state. Stem Cells 25, 1753-1760. doi: 10.1634/stemcells.2007-0068

Biswas, S., Mandal, G., Roy Chowdhury, S., Purohit, S., Payne, K. K., Anadon, C., et al. (2019). Exosomes produced by mesenchymal stem cells drive differentiation of myeloid cells into immunosuppressive M2-polarized macrophages in breast cancer. J. Immunol. Baltim. Md. 1950, 3447-3460. doi: 10.4049/jimmunol.1900692

Carreras-Planella, L., Monguió-Tortajada, M., Borràs, F. E., and Franquesa, M. (2019). Immunomodulatory effect of MSC on B cells is independent of secreted extracellular vesicles. Front. Immunol. 10:1288. doi: 10.3389/fimmu.2019.0 1288

Carvalho, A. ÉS., Sousa, M. R. R., Alencar-Silva, T., Carvalho, J. L., and SaldanhaAraujo, F. (2019). Mesenchymal stem cells immunomodulation: the road to IFN- $\gamma$ licensing and the path ahead. Cytokine Growth Factor. Rev. 47, 32-42. doi: 10.1016/j.cytogfr.2019.05.006

Chen, M., Peng, J., Xie, Q., Xiao, N., Su, X., Mei, H., et al. (2019). Mesenchymal stem cells alleviate moderate-to-severe psoriasis by reducing the production of type I interferon (IFN-I) by plasmacytoid dendritic cells ( $\mathrm{pDCs}$ ). Stem Cells Int. 2019:6961052. doi: 10.1155/2019/6961052
Despite these hardships, the capacity to adapt their immunomodulatory effects according to environmental stimuli makes MSCs superior candidates for several therapeutic approaches. Nevertheless, additional research is required to further unveil the underlying molecular mechanisms and determine optimal isolation, cell culture, and cryopreservation conditions to make MSCs meet their expectations in clinical practice.

\section{AUTHOR CONTRIBUTIONS}

LM and AT drafted the manuscript. MW, MB, RT, MB, FD, RW, and MS reviewed and edited the manuscript. All authors contributed to the article and approved the submitted version.

Chen, Q.-H., Wu, F., Liu, L., Chen, H.-B., Zheng, R.-Q., Wang, H.-L., et al. (2020). Mesenchymal stem cells regulate the Th17/Treg cell balance partly through hepatocyte growth factor in vitro. Stem Cell Res. Ther. 11:91. doi: 10.1186/ s13287-020-01612-y

Cheung, T. S., and Dazzi, F. (2018). Mesenchymal-myeloid interaction in the regulation of immunity. Semin. Immunol. 35, 59-68. doi: 10.1016/j.smim.2018. 01.002

Cho, K.-A., Lee, J.-K., Kim, Y.-H., Park, M., Woo, S.-Y., and Ryu, K.-H. (2017). Mesenchymal stem cells ameliorate B-cell-mediated immune responses and increase IL-10-expressing regulatory B cells in an EBI3-dependent manner. Cell Mol. Immunol. 14, 895. doi: 10.1038/cmi.2016.59

Cho, K.-S., Kang, S. A., Kim, S.-D., Mun, S.-J., Yu, H. S., and Roh, H.-J. (2019). Dendritic cells and M2 macrophage play an important role in suppression of Th2-mediated inflammation by adipose stem cells-derived extracellular vesicles. Stem Cell Res. 39:101500. doi: 10.1016/j.scr.2019.101500

Court, A. C., Le-Gatt, A., Luz-Crawford, P., Parra, E., Aliaga-Tobar, V., Bátiz, L. F., et al. (2020). Mitochondrial transfer from MSCs to T cells induces Treg differentiation and restricts inflammatory response. EMBO Rep. 21:e48052. doi: 10.15252/embr.201948052

Czapla, J., Matuszczak, S., Kulik, K., Wiśniewska, E., Pilny, E., Jarosz-Biej, M., et al. (2019). The effect of culture media on large-scale expansion and characteristic of adipose tissue-derived mesenchymal stromal cells. Stem Cell Res. Ther. 10:235. doi: 10.1186/s13287-019-1331-9

de Witte, S. F. H., Luk, F., Sierra Parraga, J. M., Gargesha, M., Merino, A., Korevaar, S. S., et al. (2018). Immunomodulation By therapeutic mesenchymal stromal cells (MSC) is triggered through phagocytosis of MSC by monocytic cells. Stem Cells 36, 602-615. doi: 10.1002/stem.2779

Deng, H., Wu, L., Liu, M., Zhu, L., Chen, Y., Zhou, H., et al. (2020). Bone marrow mesenchymal stem cell-derived exosomes attenuate LPSinduced ARDS by modulating macrophage polarization through inhibiting glycolysis in macrophages. Shock 54, 828-843. doi: 10.1097/SHK.000000000 0001549

Deng, Y., Zhang, Y., Ye, L., Zhang, T., Cheng, J., Chen, G., et al. (2016). Umbilical Cord-derived mesenchymal stem cells instruct monocytes towards an IL10producing phenotype by secreting IL6 and HGF. Sci. Rep. 6:37566. doi: 10.1038/ srep37566

Di Nicola, M., Carlo-Stella, C., Magni, M., Milanesi, M., Longoni, P. D., Matteucci, P., et al. (2002). Human bone marrow stromal cells suppress T-lymphocyte proliferation induced by cellular or nonspecific mitogenic stimuli. Blood 99, 3838-3843. doi: 10.1182/blood.v99.10.3838

Dominici, M., Le Blanc, K., Mueller, I., Slaper-Cortenbach, I., Marini, F. C., Krause, D. S., et al. (2006). Minimal criteria for defining multipotent mesenchymal stromal cells. The international society for cellular therapy position statement. Cytotherapy 8, 315-317. doi: 10.1080/14653240600855905

Dong, L., Chen, X., Shao, H., Bai, L., Li, X., and Zhang, X. (2018). Mesenchymal stem cells inhibited dendritic cells via the regulation of STAT1 and STAT6 phosphorylation in experimental autoimmune uveitis. Curr. Mol. Med. 17, 478-487. doi: 10.2174/1566524018666180207155614 
Engela, A. U., Hoogduijn, M. J., Boer, K., Litjens, N. H. R., Betjes, M. G. H., Weimar, W., et al. (2013). Human adipose-tissue derived mesenchymal stem cells induce functional de-novo regulatory T cells with methylated FOXP3 gene DNA. Clin. Exp. Immunol. 173, 343-354. doi: 10.1111/cei.12120

English, K., Ryan, J. M., Tobin, L., Murphy, M. J., Barry, F. P., and Mahon, B. P. (2009). Cell contact, prostaglandin $\mathrm{E}(2)$ and transforming growth factor beta 1 play non-redundant roles in human mesenchymal stem cell induction of CD4+CD25(High) forkhead box P3+ regulatory T cells. Clin. Exp. Immunol. 156, 149-160. doi: 10.1111/j.1365-2249.2009.03874.x

Feng, B., Zhu, J., Xu, Y., Chen, W., Sheng, X., Feng, X., et al. (2020). Immunosuppressive effects of mesenchymal stem cells on lung B cell gene expression in LPS-induced acute lung injury. Stem Cell Res. Ther. 11:418. doi: 10.1186/s13287-020-01934-x

François, M., Romieu-Mourez, R., Li, M., and Galipeau, J. (2012). Human MSC suppression correlates with cytokine induction of indoleamine 2,3-dioxygenase and bystander M2 macrophage differentiation. Mol. Ther. J. Am. Soc. Gene Ther. 20, 187-195. doi: 10.1038/mt.2011.189

Galleu, A., Riffo-Vasquez, Y., Trento, C., Lomas, C., Dolcetti, L., Cheung, T. S., et al. (2017). Apoptosis in mesenchymal stromal cells induces in vivo recipientmediated immunomodulation. Sci. Transl. Med. 9:eaam7828. doi: 10.1126/ scitranslmed.aam7828

Ganguly, D., Haak, S., Sisirak, V., and Reizis, B. (2013). The role of dendritic cells in autoimmunity. Nat. Rev. Immunol. 13, 566-577. doi: 10.1038/nri3477

Ge, W., Jiang, J., Arp, J., Liu, W., Garcia, B., and Wang, H. (2010). Regulatory T-cell generation and kidney allograft tolerance induced by mesenchymal stem cells associated with indoleamine 2,3-dioxygenase expression. Transplantation 90, 1312-1320. doi: 10.1097/TP.0b013e3181fed001

Guo, L., Lai, P., Wang, Y., Huang, T., Chen, X., Geng, S., et al. (2020). Extracellular vesicles derived from mesenchymal stem cells prevent skin fibrosis in the cGVHD mouse model by suppressing the activation of macrophages and B cells immune response. Int. Immunopharmacol. 84:106541. doi: 10.1016/j.intimp. 2020.106541

Guo, L., Lai, P., Wang, Y., Huang, T., Chen, X., Luo, C., et al. (2019). Extracellular vesicles from mesenchymal stem cells prevent contact hypersensitivity through the suppression of $\mathrm{Tc} 1$ and $\mathrm{Th} 1$ cells and expansion of regulatory $\mathrm{T}$ cells. Int. Immunopharmacol. 74:105663. doi: 10.1016/j.intimp.2019.05.048

He, X., Dong, Z., Cao, Y., Wang, H., Liu, S., Liao, L., et al. (2019). MSC-derived exosome promotes M2 polarization and enhances cutaneous wound healing. Stem Cells Int. 2019:7132708. doi: 10.1155/2019/7132708

Jiang, L., Zhang, S., Hu, H., Yang, J., Wang, X., Ma, Y., et al. (2019). Exosomes derived from human umbilical cord mesenchymal stem cells alleviate acute liver failure by reducing the activity of the NLRP 3 inflammasome in macrophages. Biochem. Biophys. Res. Commun. 508, 735-741. doi: 10.1016/j.bbrc.2018.11.189

Jiang, X.-X., Zhang, Y., Liu, B., Zhang, S.-X., Wu, Y., Yu, X.-D., et al. (2005). Human mesenchymal stem cells inhibit differentiation and function of monocyte-derived dendritic cells. Blood 105, 4120-4126. doi: 10.1182/blood2004-02-0586

Jin, L., Deng, Z., Zhang, J., Yang, C., Liu, J., Han, W., et al. (2019). Mesenchymal stem cells promote type 2 macrophage polarization to ameliorate the myocardial injury caused by diabetic cardiomyopathy. J. Transl. Med. 17:251. doi: 10.1186/s12967-019-1999-8

Khosravi, M., Karimi, M. H., Hossein Aghdaie, M., Kalani, M., Naserian, S., and Bidmeshkipour, A. (2017). Mesenchymal stem cells can induce regulatory $\mathrm{T}$ cells via modulating miR-126a but not miR-10a. Gene 627, 327-336. doi: 10. 1016/j.gene.2017.06.012

Kim, J., and Hematti, P. (2009). Mesenchymal stem cell-educated macrophages: a novel type of alternatively activated macrophages. Exp. Hematol 37, 1445-1453. doi: 10.1016/j.exphem.2009.09.004

Ko, J. H., Lee, H. J., Jeong, H. J., Kim, M. K., Wee, W. R., Yoon, S.-O., et al. (2016). Mesenchymal stem/stromal cells precondition lung monocytes/macrophages to produce tolerance against allo- and autoimmunity in the eye. Proc. Natl. Acad. Sci. U.S.A. 113, 158-163. doi: 10.1073/pnas.1522905113

Kozlowska, U., Krawczenko, A., Futoma, K., Jurek, T., Rorat, M., Patrzalek, D., et al. (2019). Similarities and differences between mesenchymal stem/progenitor cells derived from various human tissues. World J. Stem Cells 11, 347-374. doi: 10.4252/wjsc.v11.i6.347

Krampera, M. (2011). Mesenchymal stromal cell "licensing": a multistep process. Leukemia 25, 1408-1414. doi: 10.1038/leu.2011.108
Krampera, M., Cosmi, L., Angeli, R., Pasini, A., Liotta, F., Andreini, A., et al. (2006). Role for interferon-gamma in the immunomodulatory activity of human bone marrow mesenchymal stem cells. Stem Cells 24, 386-398. doi: 10.1634/stemcells. 2005-0008

Krampera, M., Glennie, S., Dyson, J., Scott, D., Laylor, R., Simpson, E., et al. (2003). Bone marrow mesenchymal stem cells inhibit the response of naive and memory antigen-specific T cells to their cognate peptide. Blood 101, 3722-3729. doi: 10.1182/blood-2002-07-2104

Kulkarni, M., Ozgur, S., and Stoecklin, G. (2010). On track with P-bodies. Biochem. Soc. Trans. 38, 242-251. doi: 10.1042/BST0380242

Lee, R. H., Yu, J. M., Foskett, A. M., Peltier, G., Reneau, J. C., Bazhanov, N., et al. (2014). TSG-6 as a biomarker to predict efficacy of human mesenchymal stem/progenitor cells (hMSCs) in modulating sterile inflammation in vivo. Proc. Natl. Acad. Sci. U.S.A. 111, 16766-16771. doi: 10.1073/pnas.1416121 111

Lee, S., Kim, S., Chung, H., Moon, J. H., Kang, S. J., and Park, C.-G. (2020). Mesenchymal stem cell-derived exosomes suppress proliferation of $\mathrm{T}$ cells by inducing cell cycle arrest through p27kip1/Cdk2 signaling. Immunol. Lett. 225 , 16-22. doi: 10.1016/j.imlet.2020.06.006

Leng, Z., Zhu, R., Hou, W., Feng, Y., Yang, Y., Han, Q., et al. (2020). Transplantation of ACE2- mesenchymal stem cells improves the outcome of patients with COVID-19 pneumonia. Aging Dis. 11, 216-228. doi: 10.14336/ AD. 2020.0228

Li, W., Chen, W., Huang, S., Yao, G., Tang, X., and Sun, L. (2020a). Mesenchymal stem cells prevent overwhelming inflammation and reduce infection severity via recruiting CXCR3+ regulatory T cells. Clin. Transl. Immunol. 9:e1181. doi: $10.1002 / \mathrm{cti} 2.1181$

Li, X., Dong, Y., Yin, H., Qi, Z., Wang, D., and Ren, S. (2020b). Mesenchymal stem cells induced regulatory dendritic cells from hemopoietic progenitor cells through Notch pathway and TGF- $\beta$ synergistically. Immunol. Lett. 222, 49-57. doi: 10.1016/j.imlet.2020.03.005

Li, Y.-P., Paczesny, S., Lauret, E., Poirault, S., Bordigoni, P., Mekhloufi, F., et al. (2008). Human mesenchymal stem cells license adult CD34+ hemopoietic progenitor cells to differentiate into regulatory dendritic cells through activation of the Notch pathway. J. Immunol. Baltim. Md. 1950, 1598-1608. doi: 10.4049/jimmunol.180.3.1598

Liu, F., Qiu, H., Xue, M., Zhang, S., Zhang, X., Xu, J., et al. (2019a). MSCsecreted TGF- $\beta$ regulates lipopolysaccharide-stimulated macrophage M2-like polarization via the Akt/FoxO1 pathway. Stem Cell Res. Ther. 10:345. doi: 10. 1186/s13287-019-1447-y

Liu, H., Liang, Z., Wang, F., Zhou, C., Zheng, X., Hu, T., et al. (2019b). Exosomes from mesenchymal stromal cells reduce murine colonic inflammation via a macrophage-dependent mechanism. JCI Insight 4:e131273. doi: 10.1172/jci. insight. 131273

Liu, Y., Yin, Z., Zhang, R., Yan, K., Chen, L., Chen, F., et al. (2014). MSCs inhibit bone marrow-derived DC maturation and function through the release of TSG6. Biochem. Biophys. Res. Commun. 450, 1409-1415. doi: 10.1016/j.bbrc.2014. 07.001

Lu, Z., Chang, W., Meng, S., Xu, X., Xie, J., Guo, F., et al. (2019). Mesenchymal stem cells induce dendritic cell immune tolerance via paracrine hepatocyte growth factor to alleviate acute lung injury. Stem Cell Res. Ther. 10:372. doi: $10.1186 /$ s13287-019-1488-2

Lu, Z., Meng, S., Chang, W., Fan, S., Xie, J., Guo, F., et al. (2020). Mesenchymal stem cells activate Notch signaling to induce regulatory dendritic cells in LPSinduced acute lung injury. J. Transl. Med. 18:241. doi: 10.1186/s12967-02002410- $\mathrm{z}$

Luk, F., Carreras-Planella, L., Korevaar, S. S., de Witte, S. F. H., Borràs, F. E., Betjes, M. G. H., et al. (2017). Inflammatory conditions dictate the effect of mesenchymal stem or stromal cells on B cell function. Front. Immunol. 8:1042. doi: 10.3389/fimmu.2017.01042

Luz-Crawford, P., Djouad, F., Toupet, K., Bony, C., Franquesa, M., Hoogduijn, M. J., et al. (2016). Mesenchymal stem cell-derived interleukin 1 receptor antagonist promotes macrophage polarization and inhibits B cell differentiation. Stem Cells 34, 483-492. doi: 10.1002/stem.2254

Luz-Crawford, P., Hernandez, J., Djouad, F., Luque-Campos, N., Caicedo, A., Carrère-Kremer, S., et al. (2019). Mesenchymal stem cell repression of Th17 cells is triggered by mitochondrial transfer. Stem Cell Res. Ther. 10:232. doi: $10.1186 / \mathrm{s} 13287-019-1307-9$ 
Maccario, R., Podestà, M., Moretta, A., Cometa, A., Comoli, P., Montagna, D., et al. (2005). Interaction of human mesenchymal stem cells with cells involved in alloantigen-specific immune response favors the differentiation of CD4+ T-cell subsets expressing a regulatory/suppressive phenotype. Haematologica 90, 516-525

Magatti, M., Masserdotti, A., Bonassi Signoroni, P., Vertua, E., Stefani, F. R., Silini, A. R., et al. (2020). B lymphocytes as targets of the immunomodulatory properties of human amniotic mesenchymal stromal cells. Front. Immunol. 11:1156. doi: 10.3389/fimmu.2020.01156

Malcherek, G., Jin, N., Hückelhoven, A. G., Mani, J., Wang, L., Gern, U., et al. (2014). Mesenchymal stromal cells inhibit proliferation of virus-specific CD8 + T cells. Leukemia 28, 2388-2394. doi: 10.1038/leu.2014.273

Maqbool, M., Algraittee, S. J. R., Boroojerdi, M. H., Sarmadi, V. H., John, C. M., Vidyadaran, S., et al. (2020). Human mesenchymal stem cells inhibit the differentiation and effector functions of monocytes. Innate Immun. 26, 424-434. doi: 10.1177/1753425919899132

Ménard, C., Dulong, J., Roulois, D., Hébraud, B., Verdière, L., Pangault, C., et al. (2020). Integrated transcriptomic, phenotypic, and functional study reveals tissue-specific immune properties of mesenchymal stromal cells. Stem Cells 38, 146-159. doi: 10.1002/stem.3077

Min, H., Xu, L., Parrott, R., Overall, C. C., Lillich, M., Rabjohns, E. M., et al. (2020). Mesenchymal stromal cells reprogram monocytes and macrophages with processing bodies. Stem Cells 39, 115-128. doi: 10.1002/stem.3292

Moll, G., Drzeniek, N., Kamhieh-Milz, J., Geissler, S., Volk, H.-D., and Reinke, P. (2020). MSC therapies for COVID-19: importance of patient coagulopathy, thromboprophylaxis, cell product quality and mode of delivery for treatment safety and efficacy. Front. Immunol. 11:1091. doi: 10.3389/fimmu.2020.01091

Moll, G., Geißler, S., Catar, R., Ignatowicz, L., Hoogduijn, M. J., Strunk, D., et al. (2016). Cryopreserved or fresh mesenchymal stromal cells: only a matter of taste or key to unleash the full clinical potential of MSC therapy? Adv. Exp. Med. Biol. 951, 77-98. doi: 10.1007/978-3-319-45457-3-7

Munneke, J. M., Spruit, M. J. A., Cornelissen, A. S., van Hoeven, V., Voermans, C., and Hazenberg, M. D. (2016). The potential of mesenchymal stromal cells as treatment for severe steroid-refractory acute graft-versus-host disease: a critical review of the literature. Transplantation 100, 2309-2314. doi: 10.1097/ TP.0000000000001029

Nauta, A. J., Kruisselbrink, A. B., Lurvink, E., Willemze, R., and Fibbe, W. E. (2006). Mesenchymal stem cells inhibit generation and function of both CD34+derived and monocyte-derived dendritic cells. J. Immunol. Baltim. Md. 1950, 2080-2087. doi: 10.4049/jimmunol.177.4.2080

Németh, K., Leelahavanichkul, A., Yuen, P. S. T., Mayer, B., Parmelee, A., Doi, K., et al. (2009). Bone marrow stromal cells attenuate sepsis via prostaglandin $E(2)$ dependent reprogramming of host macrophages to increase their interleukin-10 production. Nat. Med. 15, 42-49. doi: 10.1038/nm.1905

Obregon, C., Kumar, R., Pascual, M. A., Vassalli, G., and Golshayan, D. (2017). Update on dendritic cell-induced immunological and clinical tolerance. Front. Immunol. 8:1514. doi: 10.3389/fimmu.2017.01514

Ortiz-Virumbrales, M., Menta, R., Pérez, L. M., Lucchesi, O., Mancheño-Corvo, P., Avivar-Valderas, Á, et al. (2020). Human adipose mesenchymal stem cells modulate myeloid cells toward an anti-inflammatory and reparative phenotype: role of IL-6 and PGE2. Stem Cell Res. Ther 11:462. doi: 10.1186/s13287-02001975-2

Palomares Cabeza, V., Hoogduijn, M. J., Kraaijeveld, R., Franquesa, M., WitteBouma, J., Wolvius, E. B., et al. (2019). Pediatric mesenchymal stem cells exhibit immunomodulatory properties toward allogeneic $\mathrm{T}$ and $\mathrm{B}$ cells under inflammatory conditions. Front. Bioeng. Biotechnol. 7:142. doi: 10.3389/fbioe. 2019.00142

Panés, J., García-Olmo, D., Van Assche, G., Colombel, J. F., Reinisch, W., Baumgart, D. C., et al. (2016). Expanded allogeneic adipose-derived mesenchymal stem cells (Cx601) for complex perianal fistulas in Crohn's disease: a phase 3 randomised, double-blind controlled trial. Lancet 388, 1281-1290. doi: 10.1016/ S0140-6736(16)31203-X

Park, H. H., Lee, S., Yu, Y., Yoo, S. M., Baek, S. Y., Jung, N., et al. (2020). TGF$\beta$ secreted by human umbilical cord blood-derived mesenchymal stem cells ameliorates atopic dermatitis by inhibiting secretion of TNF- $\alpha$ and IgE. Stem Cells 38, 904-916. doi: 10.1002/stem.3183

Petrenko, Y., Vackova, I., Kekulova, K., Chudickova, M., Koci, Z., Turnovcova, K., et al. (2020). A comparative analysis of multipotent mesenchymal stromal cells derived from different sources, with a focus on neuroregenerative potential. Sci. Rep. 10:4290. doi: 10.1038/s41598-020-61167-z

Phinney, D. G., and Pittenger, M. F. (2017). Concise review: MSC-derived exosomes for cell-free therapy. Stem Cells 35, 851-858. doi: 10.1002/stem.2575

Qian, C., and Cao, X. (2018). Dendritic cells in the regulation of immunity and inflammation. Semin. Immunol. 35, 3-11. doi: 10.1016/j.smim.2017.12.002

Ramasamy, R., Fazekasova, H., Lam, E. W.-F., Soeiro, I., Lombardi, G., and Dazzi, F. (2007). Mesenchymal stem cells inhibit dendritic cell differentiation and function by preventing entry into the cell cycle. Transplantation 83, 71-76. doi: 10.1097/01.tp.0000244572.24780.54

Rasmusson, I., Le Blanc, K., Sundberg, B., and Ringdén, O. (2007). Mesenchymal stem cells stimulate antibody secretion in human B cells. Scand. J. Immunol. 65, 336-343. doi: 10.1111/j.1365-3083.2007.01905.x

Ritter, A., Friemel, A., Roth, S., Kreis, N.-N., Hoock, S. C., Safdar, B. K., et al. (2019). Subcutaneous and visceral adipose-derived mesenchymal stem cells: commonality and diversity. Cells 8:1288. doi: 10.3390/cells8101288

Saldanha-Araujo, F., Melgaço Garcez, E., Silva-Carvalho, A. E., and Carvalho, J. L. (2020). Mesenchymal stem cells: a new piece in the puzzle of COVID-19 treatment. Front. Immunol. 11:1563. doi: 10.3389/fimmu.2020.01563

Savage, N. D. L., Boer, T. D., Walburg, K. V., Joosten, S. A., Meijgaarden, K. V., Geluk, A., et al. (2008). Human anti-inflammatory macrophages induce Foxp3+GITR+CD25+ regulatory $\mathrm{T}$ cells, which suppress via membranebound TGF 3 -1. J. Immunol. 181, 2220-2226. doi: 10.4049/jimmunol.181.3. 2220

Schmidt, A., Zhang, X.-M., Joshi, R. N., Iqbal, S., Wahlund, C., Gabrielsson, S., et al. (2016). Human macrophages induce CD4+Foxp3+ regulatory $\mathrm{T}$ cells via binding and re-release of TGF-ß. Immunol. Cell Biol. 94, 747-762. doi: 10.1038/ icb. 2016.34

Shahir, M., Mahmoud Hashemi, S., Asadirad, A., Varahram, M., Kazempour-Dizaji, M., Folkerts, G., et al. (2020). Effect of mesenchymal stem cell-derived exosomes on the induction of mouse tolerogenic dendritic cells. J. Cell. Physiol. 235, 7043-7055. doi: 10.1002/jcp.29601

Shapouri-Moghaddam, A., Mohammadian, S., Vazini, H., Taghadosi, M., Esmaeili, S.-A., Mardani, F., et al. (2018). Macrophage plasticity, polarization, and function in health and disease. J. Cell. Physiol. 233, 6425-6440. doi: 10.1002/ jcp.26429

Song, Y., Lim, J.-Y., Lim, T., Im, K.-I., Kim, N., Nam, Y.-S., et al. (2020). Human mesenchymal stem cells derived from umbilical cord and bone marrow exert immunomodulatory effects in different mechanisms. World J. Stem Cells 12, 1032-1049. doi: 10.4252/wjsc.v12.i9.1032

Steinman, R. M., and Banchereau, J. (2007). Taking dendritic cells into medicine. Nature 449, 419-426. doi: 10.1038/nature06175

Tabera, S., Pérez-Simón, J. A., Díez-Campelo, M., Sánchez-Abarca, L. I., Blanco, B., López, A., et al. (2008). The effect of mesenchymal stem cells on the viability, proliferation and differentiation of B-lymphocytes. Haematologica 93, 1301-1309. doi: 10.3324/haematol.12857

Traggiai, E., Volpi, S., Schena, F., Gattorno, M., Ferlito, F., Moretta, L., et al. (2008). Bone marrow-derived mesenchymal stem cells induce both polyclonal expansion and differentiation of B cells isolated from healthy donors and systemic lupus erythematosus patients. Stem Cells 26, 562-569. doi: 10.1634/ stemcells.2007-0528

Vasandan, A. B., Jahnavi, S., Shashank, C., Prasad, P., Kumar, A., and Prasanna, S. J. (2016). Human mesenchymal stem cells program macrophage plasticity by altering their metabolic status via a PGE 2 -dependent mechanism. Sci. Rep. 6:38308. doi: 10.1038/srep38308

Viswanathan, S., Shi, Y., Galipeau, J., Krampera, M., Leblanc, K., Martin, I., et al. (2019). Mesenchymal stem versus stromal cells: international Society for cell \& gene therapy $\left(\right.$ ISCT $\left.^{\circledR}\right)$ mesenchymal stromal cell committee position statement on nomenclature. Cytotherapy 21, 1019-1024. doi: 10.1016/j.jcyt.20 19.08.002

Wang, D., Liu, N., Xie, Y., Song, B., Kong, S., and Sun, X. (2020a). Different culture method changing CD105 expression in amniotic fluid MSCs without affecting differentiation ability or immune function. J. Cell. Mol. Med. 24, 4212-4222. doi: $10.1111 / \mathrm{jcmm} .15081$

Wang, L.-T., Ting, C.-H., Yen, M.-L., Liu, K.-J., Sytwu, H.-K., Wu, K. K., et al. (2016). Human mesenchymal stem cells (MSCs) for treatment towards immune- and inflammation-mediated diseases: review of current clinical trials. J. Biomed. Sci. 23:76. doi: 10.1186/s12929-016-0289-5 
Wang, Q., Sun, B., Wang, D., Ji, Y., Kong, Q., Wang, G., et al. (2008). Murine bone marrow mesenchymal stem cells cause mature dendritic cells to promote T-cell tolerance. Scand. J. Immunol. 68, 607-615. doi: 10.1111/j.1365-3083.2008. 02180.x

Wang, Y., Han, B., Wang, Y., Wang, C., Zhang, H., Xue, J., et al. (2020b). Mesenchymal stem cell-secreted extracellular vesicles carrying TGF- $\beta 1$ upregulate miR-132 and promote mouse M2 macrophage polarization. J. Cell. Mol. Med. 24, 12750-12764. doi: 10.1111/jcmm.15860

Wehner, R., Wehrum, D., Bornhäuser, M., Zhao, S., Schäkel, K., Bachmann, M. P., et al. (2009). Mesenchymal stem cells efficiently inhibit the proinflammatory properties of 6-sulfo LacNAc dendritic cells. Haematologica 94, 1151-1156. doi: 10.3324/haematol.2008.001735

Weiss, A. R. R., and Dahlke, M. H. (2019). Immunomodulation by mesenchymal stem cells (MSCs): mechanisms of action of living, apoptotic, and dead MSCs. Front. Immunol. 10:1191. doi: 10.3389/fimmu.2019.01191

Yang, R., Gao, H., Chen, L., Fang, N., Chen, H., Song, G., et al. (2020). Effect of peripheral blood-derived mesenchymal stem cells on macrophage polarization and Th17/Treg balance in vitro. Regen. Ther. 14, 275-283. doi: 10.1016/j.reth. 2020.03.008
Zhang, F., Wang, C., Wen, X., Chen, Y., Mao, R., Cui, D., et al. (2020) Mesenchymal stem cells alleviate rat diabetic nephropathy by suppressing CD103+ DCs-mediated CD8+ T cell responses. J. Cell. Mol. Med. 24, 58175831. doi: $10.1111 /$ jcmm. 15250

Zhao, Y., Wang, L., Jin, Y., and Shi, S. (2012). Fas ligand regulates the immunomodulatory properties of dental pulp stem cells. J. Dent. Res. 91, 948-954. doi: 10.1177/0022034512458690

Conflict of Interest: The authors declare that the research was conducted in the absence of any commercial or financial relationships that could be construed as a potential conflict of interest.

Copyright (C) 2021 Müller, Tunger, Wobus, von Bonin, Towers, Bornhäuser, Dazzi, Wehner and Schmitz. This is an open-access article distributed under the terms of the Creative Commons Attribution License (CC BY). The use, distribution or reproduction in other forums is permitted, provided the original author(s) and the copyright owner(s) are credited and that the original publication in this journal is cited, in accordance with accepted academic practice. No use, distribution or reproduction is permitted which does not comply with these terms. 